19

\title{
Эффекты переключения в плазмонных схемах на основе тонких металлических пленок и наноструктур с повышенной фотопроводимостью
}

\author{
(С) М.Ю. Губин ${ }^{1}$, И.В. Дзедолик ${ }^{2}$, Т.В. Прохорова ${ }^{1}$, В.С. Перескоков ${ }^{2}$, А.Ю. Лексин ${ }^{1 ฯ}$ \\ ${ }^{1}$ Владимирский государственный университет им. Александра Григорьевича и Николая Григорьевича Столетовых, \\ 600000 Владимир, Россия \\ ${ }^{2}$ Крымский федеральный университет имени В.И. Вернадского, \\ 295007 Симферополь, Россия \\ ฯ e-mail: andrey_leksin@mail.ru
}

Поступила в редакцию 01.09.2021 г.

В окончательной редакции 27.10.2021 г.

Принята к публикации 27.10.2021 г.

\begin{abstract}
Рассмотрены особенности оптического управления распространением поверхностных плазмонполяритонов в плазмонных схемах на основе тонких металлических волноводов и полупроводниковых наноструктур. Предложена модель плазмонного резонатора с дополнительными элементами на основе полупроводниковых материалов, проявляющих сильную фотопроводимость. С помощью численного моделирования показана возможность переключения потока поверхностных плазмон-поляритонов в плазмонной схеме посредством включения/выключения внешнего оптического поля накачки, изменяющего вклад светоиндуцированной проводимости управляющих элементов.
\end{abstract}

Ключевые слова: поверхностные плазмон-поляритоны, металлический плазмонный волновод, фотопроводимость, плазмонный резонатор.

DOI: $10.21883 /$ OS.2022.03.52176.2700-21

\section{Введение}

Управление сигналами на оптических частотах привлекает особое внимание исследователей в последнее время в связи с появлением технологий для изготовления наноразмерных элементов плазмонных микросхем [1-11]. Элементы таких микросхем имеют размеры в несколько раз меньшие, чем длины волн сигналов с оптическими частотами. Трансляция сигналов по оптическим волокнам и обработка их в плазмонных микросхемах на тех же частотах без преобразования оптических сигналов в электрические и обратно позволит существенно поднять быстродействие схем управления и коммутации в глобальных и локальных телекоммуникационных сетях, в вычислительной технике. Отправной точкой для проектирования и реализации подобных плазмонных и гибридных оптоплазмонных схем послужили практические методы возбуждения, локализации и управления распространением поверхностных электромагнитных волн [12].

В настоящее время разрабатываются теоретические модели и реализуются экспериментально наноразмерные плазмонные элементы микросхем, такие как вентили и плазмонные полевые транзисторы [13-17], модуляторы [18], нанопреобразователи [19], логические элементы $[5,6]$, в том числе функционирующие при возбуждении плазмон-поляритонов полупроводниковыми квантовыми излучателями $[4,13,20,21]$ в сильнонелинейных режимах [22]. При этом размеры управляю- щих плазмонных схем будут иметь габариты в несколько раз меньшие, чем оптические схемы управления. Это преимущество плазмонных схем открывает широкие перспективы для реализации миниатюрной микропроцессорной техники, работающей на терагерцовых частоTax.

Имеется большое количество практических решений для управления поверхностными плазмон-поляритонами (ППП) в плазмонных схемах. Транслирование сигналов внутри плазмонной микросхемы обычно производится по волноводам конечного размера, которые размещены в диэлектрической среде $[1,3,4,23]$. При этом оси плазмонных волноводов могут быть искривлены, волноводы могут иметь конусообразные сужения и расширения, объединены в разветвители [24-28]. Вместе с тем альтернативной возможностью быстрого и эффективного управления распространением поверхностных плазмон-поляритонов может служить как применение новых фоточувствительных материалов [29, 30], так и использование плазмонно-индуцированных эффектов для управления фотопроводимостью [31] в плазмонных схемах.

Целью настоящей работы является развитие теоретической модели плазмонной схемы с оптическим управлением, а также численное моделирование эффектов переключения плазмон-поляритонного потока в плазмонных волноводах на основе тонких металлических пленок с использованием разработанных методов [32,33] и имеющихся программно-аппаратных средств [34]. Воз- 
буждаемые посредством дифракционных схем [35] сигнальные поверхностные плазмон-поляритоны в такой схеме передаются по плазмонному волноводу и поступают на вход объемного резонатора [36,37], геометрия которого настроена на деструктивную интерференцию по отношению к поступающему потоку ППП. Управление резонансом ППП поля внутри такого резонатора может быть реализовано с помощью дополнительных полупроводниковых вставок, обладающих высоким уровнем фотопроводимости $[38,39]$. В отсутствие внешнего освещения такие вставки обладают низким уровнем проводимости, что нарушает целостность резонатора и способствует беспрепятственному прохождению поля ППП сквозь систему. Напротив, включение внешней накачки приводит к значительному увеличению проводимости вставок, выталкиванию поля из резонатора с формированием утекающих мод (leakage mode) и значительному уменьшению интенсивности ППП на выходе из системы. В целом, рассматриваемые плазмонные схемы могут быть использованы как элементарные плазмонные вентили с оптическим управлением, интегрированные в состав более сложных плазмонных схем [40].

\section{Моды плоского плазмонного волновода, нагруженного диэлектриком}

Рассмотрим планарный волновод, который представляет собой структуру диэлектрик/металл/диэлектрик (ДМД), бесконечный вдоль оси х и имеющий ширину, равную $2 a$, вдоль оси $y$ (рис. 1). Полагаем, что ППП распространяются вдоль оси $x$.

В этом случае точное дисперсионное уравнение для ТМ-моды, возбуждаемой монохроматическим электромагнитным полем $\sim \exp (-i \omega t)$ с частотой $\omega$ в такой

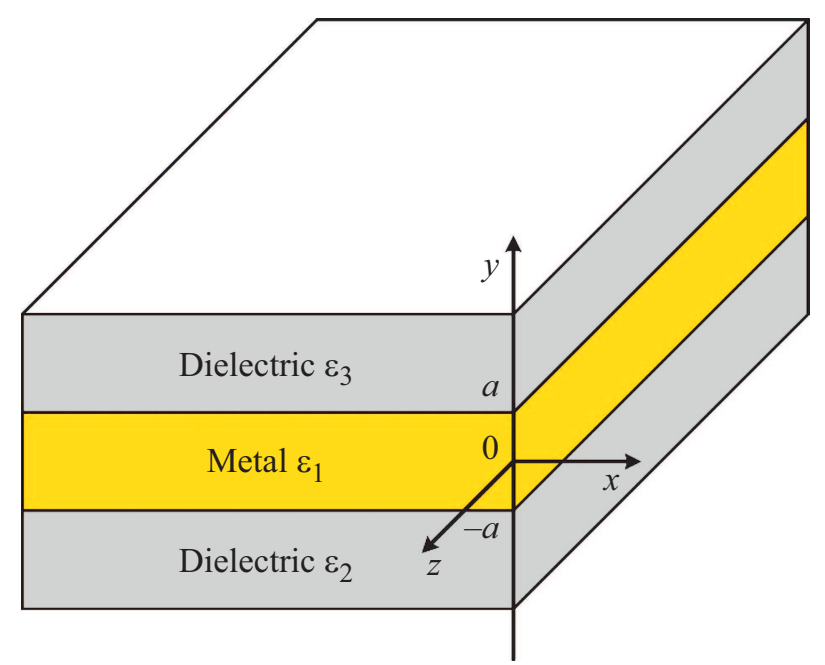

Рис. 1. Геометрия планарного волновода, бесконечного вдоль оси $x$, состоящего из тонкой металлической пленки толщиной $2 a$ с диэлектрической проницаемостью $\varepsilon_{1}$ и диэлектриков, расположенных сверху (диэлектрическая проницаемость $\varepsilon_{3}$ ) и снизу (диэлектрическая проницаемость $\varepsilon_{2}$ ) от неё. структуре, имеет следующий вид [1]:

$$
e^{-4 k_{1} a}=\frac{k_{1} / \varepsilon_{1}+k_{2} / \varepsilon_{2}}{k_{1} / \varepsilon_{1}-k_{2} / \varepsilon_{2}} \frac{k_{1} / \varepsilon_{1}+k_{3} / \varepsilon_{3}}{k_{1} / \varepsilon_{1}-k_{3} / \varepsilon_{3}},
$$

где $k_{i}=\sqrt{\beta^{2}-\varepsilon_{i} k_{0}^{2}}-$ декременты компонент моды вдоль нормальных к поверхностям волновода осей координат, $\varepsilon_{i}-$ диэлектрическая проницаемость среды, $i=1,2,3 ; \varepsilon_{0}-$ электрическая постоянная, $k_{0}=\frac{\omega}{c}-$ волновой вектор падающего излучения в вакууме, $c-$ скорость света в вакууме.

В случае, когда верхний и нижний слои имеют одинаковую диэлектрическую проницаемость $\varepsilon_{2}=\varepsilon_{3}$, дисперсионное уравнение (1) можно разбить на два уравнения [1]

$$
\begin{aligned}
& \operatorname{th} k_{1} a=-\frac{k_{2} \varepsilon_{1}}{k_{1} \varepsilon_{2}}, \\
& \operatorname{th} k_{1} a=-\frac{k_{1} \varepsilon_{2}}{k_{2} \varepsilon_{1}} .
\end{aligned}
$$

Уравнение (2а) определяет моду с поляризацией $\left(E_{x}(y)\right.$ - нечетная функция, $H_{z}(y)$ и $E_{y}(y)$ - чётные функции), тогда как уравнение (2b) определяет моду с поляризацией $\left(E_{x}(y)-\right.$ чётная функция, $H_{z}(y)$ и $E_{y}(y)$ - нечётные функции) [1].

Далее в работе рассматриваются только возбуждаемые моды ППП с чётной функцией $E_{x}(y)$. Используя приближение $\left|k_{1} a\right| \ll 1$ и полагая, что th $k_{1} a \approx k_{1} a$, можно получить приближённые аналитические решения для трансцендентного уравнения (2b), которые записываются в следующем виде:

$$
\begin{gathered}
\beta^{2}=\varepsilon_{1} k_{0}^{2}, \\
\beta^{2}=\frac{\varepsilon_{2}^{2}}{a^{2} \varepsilon_{1}^{2}}+\varepsilon_{2} k_{0}^{2} .
\end{gathered}
$$

При этом решение (3а) является тривиальным, а именно $k_{1}=0$, не зависит от толщины металлической плёнки и соответствует излучению, распространяющемуся в среде с $\varepsilon_{1}$ параллельно оси $x$, т. е. внутри металлической плёнки. Данное решение находится за рамками сделанных приближений, так как $E_{x}=0$, т. е. оно не соответствует ТМ-моде, возбуждаемой в рассматриваемом волноводе. Далее в работе рассматривается только решение (3 b). Следует отметить, что решение (3b) может быть использовано в качестве начального значения при решении трансцендентного уравнения (2b), даже если условие малости параметра разложения $\left|k_{1} a\right| \ll 1$ выполняется не строго.

\section{Управление распространением ППП в плазмонных схемах на основе плазмонных волноводов и наноструктур с высокой фотопроводимостью}

В качестве основы для управления потоками ППП в плазмонных схемах рассмотрим резонаторы, реали- 


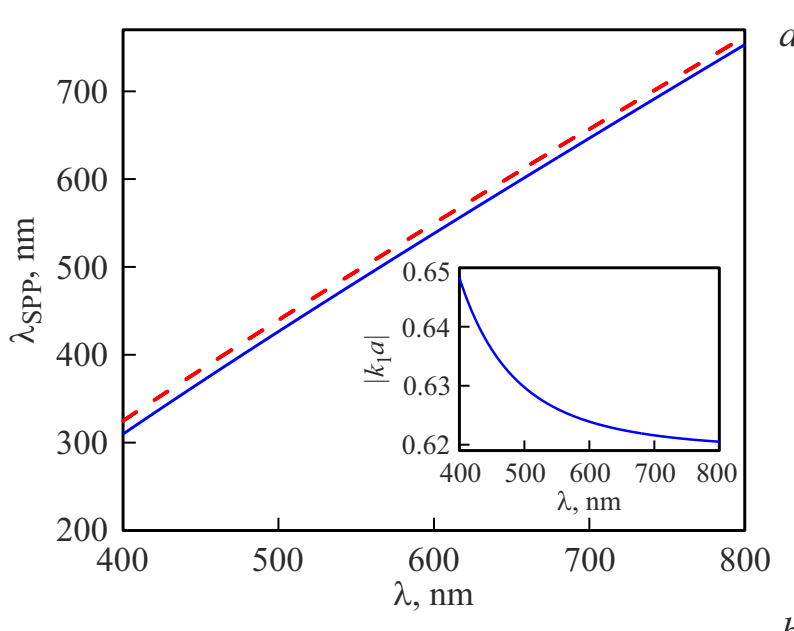

$b$

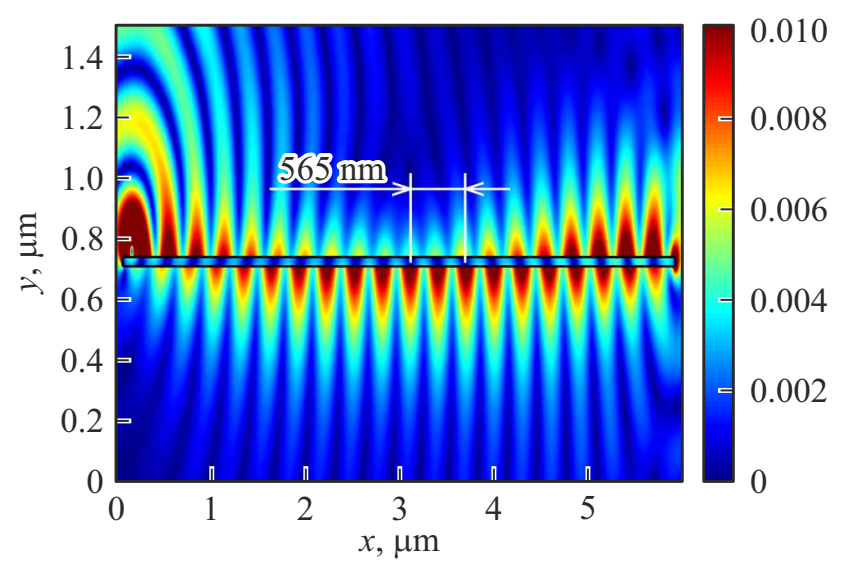

Рис. 2. (a) Зависимость длины волны ППП $\lambda_{S P P}$ от длины волны падающего излучения $\lambda$ для моды с чётной функцией $E_{x}(y)$, полученная с использованием аналитического решения $(3, b)$ (красная штриховая кривая) и численным решением трансцендентного уравнения $(2, b)$ (синяя сплошная кривая). На вставке: зависимость параметра $\left|k_{1} a\right|$ от длины волны падающего излучения $\lambda$ для моды с чётной функцией $E_{x}(y)$, полученная с использованием численного решения трансцендентного уравнения $(2, b)$. (b) Контурные зависимости для нормированного электрического поля $E=\sqrt{E_{x}^{2}+E_{y}^{2}}$, распространяющегося в тонкой золотой плёнке толщиной $2 a=30 \mathrm{~nm}$ $\left(\varepsilon_{1}=-16.12+0.23 i\right.$ для длины волны в воздухе $\left.\lambda=630 \mathrm{~nm}\right)$, которая расположена в воздухе $\left(\varepsilon_{d} \equiv \varepsilon_{2}=\varepsilon_{3}=1\right)$. Сплошные линии соответствуют расположению тонкой металлической плёнки.

зованные на основе плазмонных волноводов диэлектрик/металл/диэлектрик, поддерживающих ТМ-моды. В качестве таких волноводов могут быть задействованы тонкие металлические пленки, длина волны ППП $\lambda_{S P P}$ в которых может быть определена либо приближенно с помощью выражения (3b), либо найдена путём численного решения трансцендентного уравнения (2b). Длина волны ППП зависит от диэлектрической проницаемости $\varepsilon_{d} \equiv \varepsilon_{2}=\varepsilon_{3}$ окружающего металл диэлектрика, проводимости $\sigma$ металлической пленки и её толщины $2 a$ и рассчитывается по формуле $\lambda_{S P P}=\frac{2 \pi}{\operatorname{Re} \beta}$.
В свою очередь, высокочастотная проводимость металла $\sigma$ может быть получена на основе статической проводимости $\sigma_{S T}$ в виде $\sigma \frac{\sigma_{S T}}{1-i \omega \tau}$, где $\omega-$ соответствует частоте возбуждающего ППП поля, $\tau-$ время электронной релаксации. При этом диэлектрическая проницаемость металла записывается в виде $\varepsilon_{1}=1+i \frac{\sigma}{\omega \varepsilon_{0}}$. Статическая проводимость $\sigma_{S T}=\varepsilon_{0} \omega_{p}^{2} \tau$ зависит от электронной плазменной частоты $\omega_{p}=\sqrt{\frac{n_{e} e^{2}}{\varepsilon_{0} m_{e}}}$, где $m_{e}-$ эффективная масса, а $n_{e}-$ концентрация свободных электронов в металле. Для золота эффективная масса электрона $m_{e}=1.224 m_{0} \quad\left(m_{0}-\right.$ масса свободного электрона) и $n_{e}=5.91 \cdot 10^{28} \mathrm{~m}^{-3}, \tau=2.5 \cdot 10^{-14} \mathrm{~s}$, в соответствии с чем плазменная частота имеет величину $\omega_{p}=1.238 \cdot 10^{16} \mathrm{~s}^{-1}$, а статическая проводимость равна $\sigma_{S T}=3.39 \cdot 10^{7} \mathrm{~S} / \mathrm{m}$. Тогда при выборе тонкой золотой пленки толщиной $2 a=30 \mathrm{~nm}$ получаем длину волны ППП $\lambda_{S P P}=571 \mathrm{~nm}$ в соответствии с $(2 \mathrm{~b})$ для моды с чётной функцией $E_{x}(y)$ при условии возбуждения ППП лазерным пучком с длиной волны $\lambda=630 \mathrm{~nm}$ в воздухе.

На рис. 2, а изображены зависимости длины волны ППП $\lambda_{S P P}$ от длины волны падающего излучения $\lambda$ для плоского металлического волновода, состоящего из золотой плёнки толщиной $2 a=30 \mathrm{~nm}$, которая расположена в воздухе. Следует отметить, что максимальное расхождение между аналитическим решением (3b) и численным решением уравнения $(2 \mathrm{~b})$ составляет порядка 5\% в выбранном диапазоне длин волн падающего излучения, при том что значения параметра разложения $\left|k_{1} a\right|$ составляют порядка 0.6 (вставка на рис. 2,a). При этом длина волны ППП, полученная численным решением трансцендентного уравнения (2b), имеет величину $\lambda_{S P P}=571 \mathrm{~nm}$ (рис. 2,a). Данное значение хорошо согласуется с длиной воны ППП, равной $565 \mathrm{~nm}$, полученной с помощью численного моделирования на основе метода конечных разностей во временной области (FDTD) (рис. 2,b), реализованного авторами в программной среде MATLAB, детали расчёта представлены в Приложении А. При этом ППП возбуждаются за счёт взаимодействия электромагнитного излучения с боковыми поверхностями металлической плёнки и распространяются вдоль них.

На практике оптическое управление потоком ППП может быть реализовано при использовании плазмонного двумерного резонатора, а также управляющих наноразмерных элементов (рис. 3,a). При этом геометрия резонатора настроена на резонанс с поступающим потоком ППП, который формируется на входном порту (INPUT). При распространении поток ППП взаимодействует с выходным портом схемы (OUTPUT), на конце которого детектируется наличие или отсутствие потока ППП. Между входным и выходным портами расположен резонатор, часть которого выполнена из материала, проводимостью которого можно управлять внешним воздействием. Для осуществления быстрого оптического управления потоком ППП и смены режимов возбуждения резонатора и формирования утекающих мод удобно 

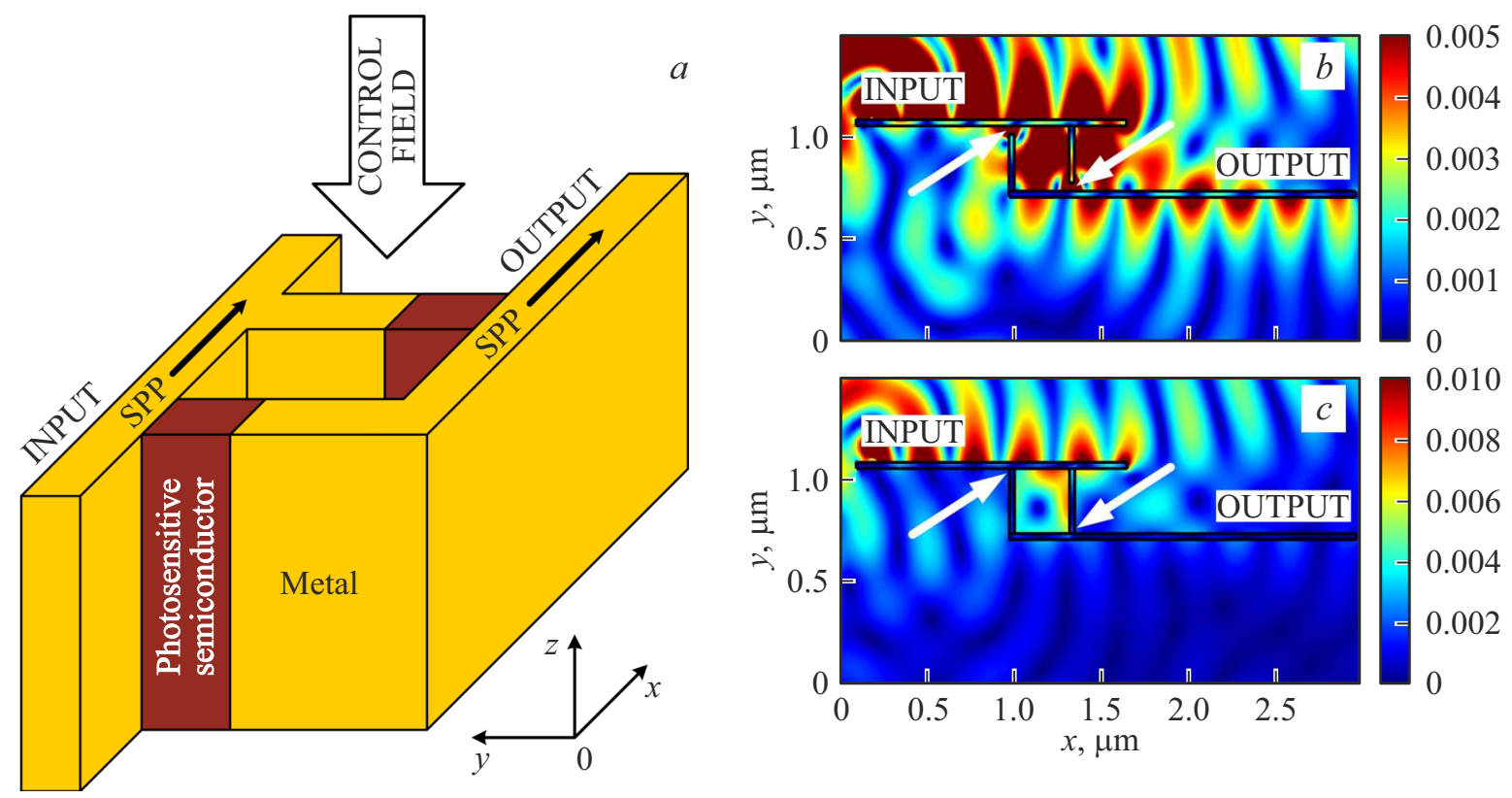

Рис. 3. (a) Схема для оптического управления потоком ППП на основе резонатора, состоящего из тонких металлических плёнок и наноразмерных вставок из фоточувствительного полупроводникового материала. Контурные зависимости для нормированного электрического поля $E=\sqrt{E_{x}^{2}+E_{y}^{2}}:(b)$ в отсутствие и $(c)$ в присутствии поля лазерной накачки. $(b),(c)$ Сплошные линии соответствуют расположению тонких металлических плёнок толщиной $2 a=30 \mathrm{~nm}\left(\varepsilon_{1}=-16.12+0.23 i\right.$ для длины волны в воздухе $\lambda=630 \mathrm{~nm})$, расположенных в воздухе $\left(\varepsilon_{d} \equiv \varepsilon_{2}=\varepsilon_{3}=1\right)$; места расположения вставок указаны стрелками. Толщина вставки $30 \mathrm{~nm}$, длина вставки $40 \mathrm{~nm}$, длина и высота резонатора (по внутренней части) составляют $315 \mathrm{~nm}$.

использовать полупроводниковые материалы с высоким уровнем фотопроводимости. К таким материалам относится карбин [41] и углеродные квантовые точки [42] и наноструктуры, электроны которых обладают высокой подвижностью $u_{e}=10^{4} \mathrm{~cm}^{2} /(\mathrm{Vs})[43,44]$ и большим средним временем жизни порядка $\tau_{l}=350 \mathrm{~ms}$ [45]. При выборе такой фоточувствительной наноразмерной вставки с концентрацией электронов $n=10^{16} \mathrm{~cm}^{-3}$ постоянная часть удельной электропроводности $\sigma_{0}=e n u_{e}$ составит $\sigma_{0}=1.6 \cdot 10^{3} \mathrm{~S} / \mathrm{m}$. Вместе с тем светоиндуцированная часть проводимости может быть представлена в виде $\Delta \sigma=\Delta \sigma_{0}\left(1-\exp \left(-t / \tau_{l}\right)\right)$, где добавка к стационарной части фотопроводимости описывается соотношением вида $\Delta \sigma_{0}=e u_{e} \Delta n$, а $\Delta n=\eta \frac{I_{0}}{h c^{2}}(h-$ постоянная Планка и $c$ - скорость света в вакууме) и представляет собой избыточную концентрацию электронов за счет фотопроводимости, где $\eta-$ эффективность генерации электронов, $I_{0}-$ интенсивность возбуждающего поля на длине волны $\lambda$. Сходную со значениями для металла проводимость можно получить при облучении фоточувствительной вставки излучением импульсного лазера на длине волны $\lambda=630 \mathrm{~nm}$ с интенсивностью $I 0=1.8 \cdot 10^{11} \mathrm{~W} / \mathrm{cm}^{2}$ при условии $\eta=0.1$.

Возможная схема эксперимента представлена на рис. 3,a. Размеры и расположение металлических плёнок, а также размеры наноразмерных вставок из фоточувствительного полупроводника подбирались с использованием численного моделирования для получе- ния необходимых режимов работы схемы. Контурные зависимости для нормированного электрического поля $E=\sqrt{E_{x}^{2}+E_{y}^{2}}$ в отсутствие и в присутствии поля лазерной накачки представлены на рис. $3, b, c$, детали расчёта представлены в Приложении А. Параметры моделирования составляют: толщина металлических плёнок $2 a=30 \mathrm{~nm}$, толщина фоточувствительной вставки $30 \mathrm{~nm}$, длина вставки $40 \mathrm{~nm}$, длина и высота резонатора (по внутренней части) составляют $315 \mathrm{~nm}$, длина волны возбуждающего лазерного пучка $\lambda=630 \mathrm{~nm}$ в воздухе $\left(\varepsilon_{d} \equiv \varepsilon_{2}=\varepsilon_{3}=1\right)$, диэлектрическая проницаемость золота на длине воны $\lambda=630 \mathrm{~nm}$ составит $\varepsilon_{1}=-16.12+0.23 i$.

Полагаем, что оптическое управление плазмонной схемой осуществляется путем включения/выключения поля лазерной накачки, что приводит к изменению проводимости фоточувствительной вставки. В отсутствие поля накачки, когда проводимость фоточувствительной вставки соответствует $\sigma_{0}$, поток ППП свободно проходит через резонатор (рис. $3, b$ ). Включение поля накачки на длине волны $\lambda$ вызывает увеличение проводимости материала до уровня $\sigma_{0}+\Delta \sigma_{0}$, что приводит к реализации резонансных условий деструктивной интерференции, выталкиванию поля из резонатора и формированию режима утекающих мод. Это влечет за собой значительное уменьшение интенсивности потока ППП, пропускаемого плазмонной схемой (рис. 3,c). 
На основе данных численного моделирования могут быть получены оценки для величины контраста переключения $V=\frac{I_{O F F}-I_{O N}}{I_{O N}}$, которая составляет $63 \%$ для случая, представленного на рис. $3, b, c$, где $I_{O N}$ и $I_{O F F}-$ интенсивности поля ППП на выходе резонатора при включенном и выключенном поле оптической накачки соответственно. Вместе с тем дополнительной проблемой практического использования представленной плазмонной схемы является небольшая временная задержка обратного переключения системы при выключении поля оптической накачки, что ограничивает скорость переключения потока ППП.

Отдельного внимания заслуживает использование эффективных ближнепольных источников ППП для рассматриваемых систем. В качестве таких источников могут использоваться как классические ближнепольные источники на основе материалов с большим показателем преломления [46], так и чисто диэлектрические наноструктуры [47], а также гибридные системы [48] для эффективного преобразования оптического излучения в поверхностные электромагнитные возбуждения на наномасштабе. При использовании ближнепольных коллективных эффектов [49] такие системы могут быть применены в качестве мощной ближнепольной накачки $[50,51]$, а также интегрированы внутрь плазмонных схем. Использование комбинированных плазмонных схем с оптическим управлением представляет интерес для разработки оптоэлектронных систем с малым временем отклика, а также сверхбыстрых сенсоров на их основе.

\section{Заключение}

В работе на основе теоретического анализа проведено изучение процессов возбуждения и распространения, а также динамики поверхностных плазмон-поляритонов в волноводных плазмонных схемах с оптическим управлением. Исследование особенностей управления потоками ППП с помощью внешнего излучения в таких схемах актуально для проектирования и создания наноразмерных оптоплазмонных вентильных элементов типа плазмонных транзисторов с возможностью их интегрирования в устройства плазмонной схемотехники для быстрого многорежимного управления и многонаправленного переключения электромагнитных сигналов оптических частот. Развитие этого направления также связано с проектированием и реализаций функциональных компьютерных симуляторов субволновых плазмонных схем, предназначенных для обработки информации на оптических частотах. Моделирование наноразмерных плазмонных элементов проведено с целью дальнейшего экспериментального исследования плазмонных схем для устройств обработки информации, функционирующих на оптических частотах.

\section{Приложение A}

Для среды с дисперсией (зависимостью параметров от частоты) метод конечных разностей во временной области (FDTD) основывается на использовании эквивалентных уравнений Максвелла [52]

$$
\begin{gathered}
\frac{\partial \mathbf{D}}{\partial t}=\nabla \times \mathbf{H}, \\
\frac{\partial \mathbf{H}}{\partial t}=-\frac{1}{\mu} \nabla \times \mathbf{E}, \\
\mathbf{D}(\omega)=\varepsilon_{0} \varepsilon(\omega) \mathbf{E}(\omega),
\end{gathered}
$$

где $\varepsilon(\omega)$ - относительная диэлектрическая проницаемость среды, D - электрическая индукция, $\mathbf{E}-$ напряженность электрического поля, $\mathbf{H}$ - напряженность магнитного поля, $\mu$ - магнитная проницаемость среды, $\varepsilon_{0}$ - электрическая постоянная.

В двумерном случае (2-D FDTD модель), следуя [53], рассмотрим режим ТМ-моды, распространяющейся в направлении $x$. Проводящий однородный лист бесконечной длины расположен в плоскости $x z$. Аналогично однородными вдоль оси $z$ являются свойства остальных компонентов системы. Переходя к нормированным векторам $\tilde{\mathbf{E}}=\sqrt{\frac{\varepsilon_{0}}{\mu_{0}}} \mathbf{E}$ и $\tilde{\mathbf{D}}=\frac{1}{\sqrt{\varepsilon_{0} \mu_{0}}} \mathbf{D}$, уравнения Максвелла в покомпонентной форме примут вид

$$
\begin{gathered}
\frac{\partial \tilde{D}_{x}}{\partial t}=c \frac{\partial H_{z}}{\partial y}, \\
\frac{\partial \tilde{D}_{y}}{\partial t}=-c \frac{\partial H_{z}}{\partial x}, \\
\frac{\partial H_{z}}{\partial t}=c\left(\frac{\partial \tilde{E}_{x}}{\partial y}-\frac{\partial \tilde{E}_{y}}{\partial x}\right),
\end{gathered}
$$

где $c$ - скорость света в вакууме, $\mu_{0}-$ магнитная постоянная. При дискретизации уравнений Максвелла с использованием схемы Йи (Yee's scheme) [54] и шагом $\Delta t=\Delta z /(2 c)$, где $\Delta z$ - пространственный размер, могут быть получены основные расчетные уравнения для двумерной модели в форме

$$
\begin{aligned}
& \tilde{D}_{x}^{n+1 / 2}\left(i, j+\frac{1}{2}\right)=\tilde{D}_{x}^{n-1 / 2}\left(i, j+\frac{1}{2}\right) \\
& +\frac{1}{2}\left[H_{z}^{n}(i, j+1)-H_{z}^{n}(i, j)\right], \\
& \tilde{D}_{y}^{n+1 / 2}\left(i+\frac{1}{2}, j\right)=\tilde{D}_{y}^{n-1 / 2}\left(i+\frac{1}{2}, j\right) \\
& +\frac{1}{2}\left[H_{z}^{n}(i, j)-H_{z}^{n}(i+1, j)\right], \\
& H_{z}^{n+1}(i, j)=H_{z}^{n}(i, j) \\
& +\frac{1}{2}\left[\tilde{E}_{x}^{n+\frac{1}{2}}\left(i, j+\frac{1}{2}\right)-\tilde{E}_{x}^{n+\frac{1}{2}}\left(i, j-\frac{1}{2}\right)\right. \\
& \left.-\tilde{E}_{y}^{n+\frac{1}{2}}\left(i+\frac{1}{2}, j\right)+\tilde{E}_{y}^{n+\frac{1}{2}}\left(i-\frac{1}{2}, j\right)\right] .
\end{aligned}
$$


Система уравнений (A1) представляет собой основу для программной реализации метода FDTD.

Электрическое поле вычисляется по формуле $\tilde{\mathbf{D}}(\omega)=\varepsilon(\omega) \tilde{\mathbf{E}}(\omega)$. Объединяя это уравнение и выражение для диэлектрической проницаемости в виде разложения в полином $\varepsilon=1+\frac{\sigma_{S T}}{i \omega \varepsilon_{0}}-\frac{B}{(1+i \omega \tau) \varepsilon_{0}}$, где $B=\tau \sigma_{S T} / \varepsilon_{0}$, получаем

$$
\begin{aligned}
& \tilde{D}_{x}(\omega)=\left\{1+\frac{\sigma_{S T}}{i \omega \varepsilon_{0}}-\frac{B}{1+i \omega \tau}\right\} \tilde{E}_{x}(\omega), \\
& \tilde{D}_{y}(\omega)=\left\{1+\frac{\sigma_{S T}}{i \omega \varepsilon_{0}}-\frac{B}{1+i \omega \tau}\right\} \tilde{E}_{y}(\omega),
\end{aligned}
$$

где $\tau-$ время рассеяния электронов и $\sigma_{S T}-$ статическая проводимость. Статическая проводимость зависит от типа используемого материала, при этом для тонких металлических пленок необходимо учитывать эффекты размерного квантования и результаты экспериментальных исследований [55].

Зависимость уравнений (A2) от частоты $\omega$ можно преобразовать во временную зависимость и получить следующий набор уравнений для вычисления напряжённости электрического поля:

$$
\begin{gathered}
\tilde{E}_{x, y}^{n+1 / 2}\left(i+\frac{1}{2}, j\right) \\
=\frac{+e^{-\Delta t / \tau} S_{(x, y}^{n-1 / 2}\left(i+\frac{1}{2}, j\right)}{M\left(i+\frac{1}{2}, j\right)}, \\
I_{x, y}^{n+1 / 2}\left(i+\frac{1}{2}, j\right)=I_{x, y}^{n-1 / 2}\left(i+\frac{1}{2}, j\right) \\
+N\left(i+\frac{1}{2}, j\right) \tilde{E}_{x, y}^{n+1 / 2}\left(i+\frac{1}{2}, j\right), \\
S_{x, y}^{n+1 / 2}\left(i+\frac{1}{2}, j\right)=S_{x, y}^{n-1 / 2}\left(i+\frac{1}{2}, j\right) e^{-\Delta t / \tau} \\
+L\left(i+\frac{1}{2}, j\right) \tilde{E}_{x, y}^{n+1 / 2}\left(i+\frac{1}{2}, j\right),
\end{gathered}
$$

где

$$
\begin{aligned}
& M\left(i+\frac{1}{2}, j\right)=\sigma_{S T}\left(i+\frac{1}{2}, j\right) \Delta t / \varepsilon_{0} \\
& -\Delta t B\left(i+\frac{1}{2}, j\right) / \tau\left(i+\frac{1}{-2}, j\right), \\
& N\left(i+\frac{1}{2}, j\right)=\sigma_{S T}\left(i+\frac{1}{2}, j\right) \Delta t / \varepsilon_{0}
\end{aligned}
$$

и

$$
L\left(i+\frac{1}{2}, j\right)=\Delta t B\left(i+\frac{1}{2}, j\right) / \tau\left(i+\frac{1}{2}, j\right) .
$$

\section{Финансирование работы}

Исследование выполнено при поддержке Российского научного фонда (грант № 19-72-20154).

\section{Конфликт интересов}

Авторы заявляют, что у них нет конфликта интересов.

\section{Список литературы}

[1] С.А. Майер. Плазмоника: теория и приложения (Регулярная и хаотическая динамика, Москва-Ижевск, 2011) [S.A. Maier. Plasmonics: Fundamental and Applications (Springer Science+Bussines Media, New York, 2007)].

[2] T.W. Ebbesen, C. Genet, S.I. Bozhevolnyi. Phys. Today, 61 (5), 44 (2008). DOI:10.1063/1.2930735

[3] В.В. Климов. Наноплазмоника (ФИЗМАТЛИТ, Москва, 2010) [V.V. Klimov. Nanoplasmonics (Taylor \& Francis Group, 2013). DOI: 10.1201/b15442]

[4] D.K. Gramotnev, S.I. Bozhevolnyi. Nat. Photon., 4, 83 (2010). DOI: 10.1038/nphoton.2009.282

[5] I.V. Dzedolik. Solitons and nonlinear waves of phononpolaritons and plasmon-polaritons (Nova Science Publishers, New York, 2016).

[6] T.J. Davis, D.E. Gómez, A. Roberts. Nanophotonics, 6 (3), 543 (2017). DOI: 10.1515/nanoph-2016-0131

[7] D. Xu, X. Xiong, L. Wu, X.-F. Ren, C.E. Png, G.-C. Guo, Q. Gong, Y.-F. Xiao. Adv. Opt. Phot., 10 (4), 703 (2018). DOI: $10.1364 /$ AOP. 10.000703

[8] I.P. Radko, S.I. Bozhevolnyi, A.B. Evlyukhin, A. Boltasseva. Opt. Express, 15 (11), 6576 (2007). DOI: 10.1364/OE.15.006576

[9] A. Barreda, S. Hell, M.A. Weissflog, A. Minovich, T. Pertsch, I. Staude. J. Quant. Spectrosc. Radiat. Transf., 276, 107900 (2021). DOI: 10.1016/j.jqsrt.2021.107900

[10] V.A. Zenin, S.I. Bozhevolnyi. Nanophotonics, 10 (14), 3613 (2021). DOI: 10.1515/nanoph-2021-0267

[11] J. Lee, D.-J. Jeon, J.-S. Yeo. Adv. Mater., (in print) 2006606 (2021). DOI: 10.1002/adma.202006606

[12] S.I. Bozhevolnyi, V.S. Volkov, E. Devaux, T.W. Ebbesen. Phys. Rev. Lett., 95 (4), 046802 (2005). DOI: 10.1103/PhysRevLett.95.046802

[13] I.A. Pshenichnyuk, S.S. Kosolobov, V.P. Drachev. Appl. Sci., 9 (22), 4834 (2019). DOI: 10.3390/app9224834

[14] D.E. Chang, A.S. Sørensen, E.A. Demler, M.D. Lukin. Nat. Phys., 3, 807 (2007). DOI: 10.1038/nphys 708

[15] T. Aihara, A. Takeda, M. Fukuhara, Y. Ishii, M. Fukuda. Proc. SPIE, 8923, 89234F (2013). DOI: 10.1117/12.2033618

[16] I.V. Dzedolik, A.Yu. Leksin. J. Opt., 22 (7), 075001 (2020). DOI: $10.1088 / 2040-8986 / \mathrm{ab} 9511$

[17] I.V. Dzedolik, S. Skachkov. J. Opt. Soc. Am. A, 36 (5), 775 (2019). DOI: 10.1364/JOSAA.36.000775

[18] C. Hoessbacher, A. Josten, B. Baeuerle, Y. Fedoryshyn, H. Hettrich, Y. Salamin, W. Heni, C. Haffner, C. Kaiser, R. Schmid, D.L. Elder, D. Hillerkuss, M. Möller, L.R. Dalton, J. Leuthold. Opt. Express, 25 (3), 1762 (2017). DOI: 10.1364/OE.25.001762

[19] A.B. Evlyukhin, G. Brucoli, L. Martín-Moreno, S.I. Bozhevolnyi, F.J. García-Vidal. Phys. Rev. B, 76 (7), 075426 (2007). DOI: $10.1103 /$ PhysRevB.76.075426

[20] H. Siampour, S. Kumar, S.I. Bozhevolnyi. ACS Photonics, 4 (8), 1879 (2017). DOI: 10.1021/acsphotonics.7b00374

[21] К.А. Магарян, К.Р. Каримуллин, И.А. Васильева, А.В. Наумов. Опт. и спектр., 126 (1), 50 (2019). DOI:10.21883/OS.2019.01.47052.283-18 
[K.A. Magaryan, K.R. Karimullin, I.A. Vasil'eva, A.V. Naumov. Opt. Spectrosc., 126 (1), 41 (2019). DOI: $10.1134 / \mathrm{S} 0030400 \mathrm{X} 19010107]$.

[22] А.И. Маймистов, Е.И. Ляшко. Опт. и спектр., 126 (5), 578 (2019). DOI:10.21883/OS.2019.05.47656.356-18

[A.I. Maimistov, E.I. Lyashko. Opt. Spectrosc., 126 (5), 497 (2019). DOI: 10.1134/S0030400X19050205].

[23] P. Berini. Opt. Express, 7 (10), 329 (2000). DOI: $10.1364 /$ OE.7.000329

[24] A.V. Krasavin, A.V. Zayats. Phys. Rev. B, 78 (4), 045425 (2008). DOI: 10.1103/PhysRevB.78.045425

[25] X.-Y. Zhang, A. Hu, J. Z. Wen, T. Zhang, X.-J. Xue, Y. Zhou, W.W. Duley. Opt. Express, 18 (18), 18945 (2010). DOI: $10.1364 /$ OE.18.018945

[26] A. Kumar, J. Gosciniak, V.S. Volkov, S. Papaioannou, D. Kalavrouziotis, K. Vyrsokinos, J.-C. Weeber, K. Hassan, L. Markey, A. Dereux, T. Tekin, M. Waldow, D. Apostolopoulos, H. Avramopoulos, N. Pleros, S.I. Bozhevolnyi. Laser Photonics Rev., 7 (6), 938 (2013).

DOI: $10.1002 /$ lpor.201200113

[27] V.A. Zenin, R. Malureanu, I.P. Radko, A.V. Lavrinenko, S.I. Bozhevolnyi. Opt. Express, 24 (5), 4582 (2016). DOI: $10.1364 /$ OE.24.004582

[28] A. Said, K.S.R. Atia, S.S.A. Obayya. J. Opt. Soc. Am. B, 37 (11), A163 (2020). DOI: 10.1364/JOSAB.399121

[29] G.A. Ermolaev, D.V. Grudinin, Y.V. Stebunov, K.V. Voronin, V.G. Kravets, J. Duan, A.B. Mazitov, G.I. Tselikov, A. Bylinkin, D.I. Yakubovsky, S.M. Novikov, D.G. Baranov, A.Y. Nikitin, I.A. Kruglov, T. Shegai, P. Alonso-González, A.N. Grigorenko, A.V. Arsenin, K.S. Novoselov, V.S. Volkov. Nat. Commun., 12, 854 (2021). DOI: $10.1038 / \mathrm{s} 41467-021-21139-\mathrm{x}$

[30] A. George, M.V. Fistul, M. Gruenewald, D. Kaiser, T. Lehnert, R. Mupparapu, C. Neumann, U. Hübner, M. Schaal, N. Masurkar, L.M.R. Arava, I. Staude, U. Kaiser, T. Fritz, A. Turchanin. npj 2D Mater. Appl., 5, 15 (2021). DOI: $10.1038 / \mathrm{s} 41699-020-00182-0$

[31] E.V. Vaschenko, T.A. Vartanyan, V.V. Khromov, N.B. Leonov, S.G. Przhibelskii, F. Hubenthal. Proc. SPIE, 7996, 79960K (2011). DOI: $10.1117 / 12.887582$

[32] M.Yu. Gubin, A.Yu. Leksin, A.V. Shesterikov, V.S. Volkov, A.V. Prokhorov. Appl. Surf. Sci., 506, 144814 (2020). DOI: $10.1016 /$ j.apsusc.2019.144814

[33] M.Yu. Gubin, A.Yu. Leksin, A.V. Shesterikov, A.V. Prokhorov, V.S. Volkov. Nanomaterials, 10 (1), 122 (2020). DOI: $10.3390 /$ nano 10010122

[34] А.В. Шестериков, А.Ю. Лексин, Т.В. Прохорова, Н.М. Воронова, А.В. Прохоров. Изв. РАН. Сер. физ., 84 (3), 418 (2020). DOI: $10.31857 / \mathrm{S} 0367676520030242$

[A.V. Shesterikov, A.Y. Leksin, T.V. Prokhorova, N.M. Voronova, A.V. Prokhorov. Bull. Russ. Acad. Sci. Phys., 84 (3), 324 (2020). DOI: 10.3103/S106287382003020X].

[35] В.Б. Новиков, А.М. Ромашкина, Д.А. Езенкова, И.А. Родионов, К.Н. Афанасьев, А.В. Барышев, Т.В. Мурзина. Опт. и спектр., 128 (9), 1369 (2020).

DOI: $10.21883 / O S .2020 .09 .49878 .98-20$

[V.B. Novikov, A.M. Romashkina, D.A. Ezenkova, I.A. Rodionov, K.N. Afanas'ev, A.V. Baryshev, T.V. Murzina. Opt. Spectrosc., 128 (9), 1481 (2020). DOI: $10.1134 / \mathrm{S} 0030400 \mathrm{X} 20090155]$.

[36] Л.А. Вайнштейн. Электромагнитные волны, 2 изд. (Радио и связь, Москва, 1988).
[37] Дж. Джексон. Классическая электродинамика (Мир, Москва, 1965) [J. Jackson. Classical Electrodynamics (John Wiley \& Sons, New York, 1962)].

[38] R.L. Petritz. Phys. Rev., 104 (6), 1508 (1956). DOI:10.1103/PhysRev.104.1508

[39] M. Freitag, Y. Martin, J.A. Misewich, R. Martel, Ph. Avouris. Nano Lett., 3 (8), 1067 (2003). DOI: 10.1021/n1034313e

[40] S.I. Bozhevolnyi. Plasmonic Nanoguides and Circuits (Pan Stanford Publishing, Singapore, 2009).

[41] V.I. Kasatochkin, A.M. Sladkov, et al. Dokl. Akad. Nauk SSSR, 177 (2), 358 (1967).

[42] S. Zhu, Q. Meng, L. Wang, J. Zhang, Y. Song, H. Jin, K. Zhang, H. Sun, H. Wang, B. Yang. Angew. Chem. Int. Ed., 52 (14), 3953 (2013). DOI: 10.1002/anie.201300519

[43] W. Kwon, S. Do, D.C. Won, S.-W. Rhee. ACS Appl. Mater. Interfaces, 5 (3), 822 (2013). DOI: 10.1021/am3023898

[44] T. Dürkop, S.A. Getty, E. Cobas, M.S. Fuhrer. Nano Lett., 4 (1), 35 (2004). DOI: 10.1021/n1034841q

[45] T. Yuan, T. Meng, T. He, Y. Shi, Y. Li, X. Li, L. Fan, S. Yang. J. Mater. Chem. C, 7 (23), 6820 (2019). DOI: $10.1039 / \mathrm{C} 9 \mathrm{TC} 01730 \mathrm{E}$

[46] P.D. Terekhov, K.V. Baryshnikova, A.S. Shalin, A. Karabchevsky, A.B. Evlyukhin. Opt. Lett., 42 (4), 835 (2017). DOI: $10.1364 /$ OL. 42.000835

[47] I.S. Sinev, F.E. Komissarenko, I.S. Mukhin, M.I. Petrov, I.V. Iorsh, P.A. Belov, A.K. Samusev. Nanosyst. Phys. Chem. Math., 9 (5), 609 (2018). DOI: $10.17586 / 2220-8054-2018-9-5-609-613$

[48] J. Ho, J. Tatebayashi, S. Sergent, C.F. Fong, S. Iwamoto, Y. Arakawa. ACS Photonics, 2 (1), 165 (2015). DOI: $10.1021 / \mathrm{ph} 5003945$

[49] M.Yu. Gubin, A.V. Shesterikov, A.V. Prokhorov, V.S. Volkov. Laser Photonics Rev., 14 (12), 2000237 (2020). DOI: $10.1002 /$ por.202000237

[50] А.Г. Баканов, Н.А. Торопов, Т.А. Вартанян. Опт. и спектр., 120 (3), 502 (2016). DOI:10.7868/S0030403416030041

[A.G. Bakanov, N.A. Toropov, T.A. Vartanyan. Opt. Spectrosc., 120 (3), 477 (2016). DOI: 10.1134/S0030400X16030048]

[51] А.В. Шестериков, М.Ю. Губин, С.Н. Карпов, А.В. Прохоров. Письма в ЖЭТФ, 107 (7), 459 (2018).

DOI: $10.7868 / \mathrm{S} 0370274 \mathrm{X} 1807010 \mathrm{X}$

A.V. Shesterikov, M.Yu. Gubin, S.N. Karpov, A.V. Prokhorov. JETP Lett., 107 (7), 435 (2018). DOI: $10.1134 / \mathrm{S} 0021364018070081]$

[52] P.C. Sarker, Md.M. Rana, A.K. Sarkar. Optik, 144, 1 (2017). DOI: 10.1016/j.ijleo.2017.06.054

[53] A. Taflove, S.C. Hagness. Computational Electrodynamics: The Finite-Difference Time-Domain Method, 3rd ed. (Artech House, Boston, London, 2005).

[54] D.M. Sullivan. Electromagnetic Simulation Using the FDTD Method (IEEE Press, New York, 2000).

[55] D.I. Yakubovsky, A.V. Arsenin, Yu.V. Stebunov, D.Yu. Fedyanin, V.S. Volkov. Opt. Express, 25 (21), 25574 (2017). DOI: $10.1364 /$ OE.25.025574 